\title{
Asymptotic analysis of a planar waveguide perturbed by a non periodic perforation
}

\author{
Giuseppe Cardone ${ }^{1, a}$, Tiziana Durante $e^{2, b}$ \\ ${ }^{1}$ Universita di Napoli "Federico II", Napoli, Italy \\ ${ }^{2}$ University of Salerno, 84084 Fisciano (SA), Italy \\ ${ }^{a}$ giuseppe.cardone@unina.it, ${ }^{b}$ tdurante@unisa.it
}

Corresponding author: Giuseppe Cardone, giuseppe.cardone@unina.it

ABSTRACT We consider a general second order elliptic operator in a planar waveguide perforated by small holes distributed along a curve and subject to classical boundary conditions on the holes. Under weak assumptions on the perforation, we describe all possible homogenized problems.

KEYWORDS perforation, elliptic operator, unbounded domain, homogenization, norm resolvent convergence.

FOR CITATION Cardone G., Durante T. Asymptotic analysis of a planar waveguide perturbed by a non periodic perforation. Nanosystems: Phys. Chem. Math., 2022, 13 (1), 5-11.

\section{Introduction}

In this paper, we consider a second order elliptic operator in a planar strip perforated along a curve which can be either infinite or finite and closed. The shapes and the distribution of the holes can be rather arbitrary and no periodicity is assumed. We impose on the boundary of the holes classical boundary conditions, i.e., Dirichlet, Neumann or Robin conditions: boundaries of different holes can be subject to different types of boundary conditions.

Our operator describes a quantum particle in a non-isotropic waveguide (the infinite strip) since the coefficients of the operator are variable. The perforation represents small defects distributed along a given line, while the conditions on the boundaries of the holes impose certain regime, for instance, the Dirichlet condition describes a wall and the particle can not pass through such boundary. Our aim is to describe all possible homogenized problems. In fact, the homogenization process describes the effective behavior of our model once the perforation becomes finer, while the type of resolvent convergence characterizes in which sense the perturbed model is close to the effective one. The results we will state, are proved in [1] .

Our first main result describes the homogenized problems depending on the geometry, sizes, and distribution of the holes as well as of the conditions on the boundary of the holes. The differential expression for the homogenized operator is the same as for the original operator, but with Dirichlet condition or delta-interaction or no condition on the reference curve along which the perforation is made. Our second main result is the norm resolvent convergence of the perturbed operator to the homogenized one and the estimates for the rates of convergence. In all cases except one the operator norm is that of the operators from $L_{2}$ into $W_{2}^{1}$, while in the exceptional case it is from $L_{2}$ into $L_{2}$. Nevertheless, in the latter case we show that by employing a special boundary corrector one can replace the norm by that of the operators acting from $L_{2}$ into $W_{2}^{1}$. Such kind of results on norm resolvent convergence are completely new for the domains perforated periodically along curves or manifolds, especially in view of the fact that we succeeded in studying the general non-periodic perforation with arbitrary boundary conditions.

Our technique is based on the variational formulations of the equations for the perturbed and the homogenized operators. We use no smoothing operator like in previous papers on the operators with rapidly oscillating coefficients. Instead, we write the integral identity for the difference of the perturbed and homogenized resolvents and then estimate the terms coming from the boundary conditions. It requires certain accurate estimates for various boundary integrals over holes and over the reference curve. The main difference of our technique with that in the previous works is the assumptions for the perforation. In previous works [2-8], the main assumption was the existence of an operator of continuation the holes for the functions defined outside as well as uniform estimates for this operator. In our work, we assume the solvability of a certain fixed boundary value problems for the divergence operator in the vicinity of the holes. We believe that our assumptions are not worse than the existence of the continuation operator, since we require just a solvability of certain boundary value problem while the existence of the continuation operator means the possibility to extend each function in a given Sobolev space.

The attention to the norm resolvent convergence started a new direction in the homogenization theory. It was found for the operators with fast periodically oscillating coefficients that their resolvents converge to the resolvents of the homogenized operators in the norm resolvent sense. This was a much stronger result in comparison with known classical results stating just a weak or a strong resolvent convergence. Results based on norm resolvent convergence were obtained by M.Sh. Birman, T.A. Suslina [9-14], V.V. Zhikov and S.E. Pastukhova [15-20], G. Griso [21, 22], and by C.E. Kenig,

(C) Cardone G., Durante T., 2022 
F. Lin, Z. Shen [23,24]; see also other papers by these authors. Moreover, in the above cited works, the authors succeeded in establishing sharp estimates for the rates of convergence in the sense of various operator norms.

In view of the above described results, a natural question appeared: whether a similar norm resolvent convergence is valid for other types of the perturbations in the homogenization theory? This issue was studied recently for certain perturbations in the boundary homogenization.

In [25-33], [34-40] problems with frequent alternation of boundary conditions were treated. The norm resolvent convergence was proven for all possible homogenized problems as well as for both periodic and non-periodic alternations. Estimates for the rate of convergence were obtained. In periodic cases, certain asymptotic expansions for the spectra of perturbed operators were constructed.

In [41, 42], [43, Ch. III, Sec. 4], the norm resolvent convergence for problems with a fast periodically oscillating boundary was proven. The most general results were obtained in [41]. Namely, various geometries of oscillations as well as various boundary conditions on the oscillating boundary were considered. Estimates were obtained for the rate of norm resolvent convergence in the sense of various operator norms.

The norm resolvent convergence for periodic perforations was studied in [16,44,45] . In [44] the whole of a domain was perforated. The operator was described by the Helmholtz equation; on the boundaries of the holes the Dirichlet condition was imposed. The authors treated the case when the holes disappeared under the homogenization and made no influence for the homogenized operators. The norm resolvent convergence was proven; no estimates for the rate of convergence were found. In [16], an elliptic operator in a perforated domain was studied. Here, again, the whole of the domain was perforated. It was assumed that the sizes of the holes and the distances between them are of the same order of smallness. On the boundaries of the holes the Neumann condition was imposed. The norm resolvent convergence and the estimates for the rate of convergence were established.

One more interesting paper devoted to norm resolvent convergence is [19]. Here, the perturbation was defined by rescaling an abstract periodic measure. The main result is the description of the homogenized operator, the proof of the norm resolvent convergence, and the estimates for the rate of convergence. A general model of [19] covered various perturbations, including periodic perforation of the whole of a domain, provided the sizes of the holes and the distances between them are of the same smallness order.

\section{Problem and main results}

Let $x=\left(x_{1}, x_{2}\right)$ be the Cartesian coordinates in $\mathbb{R}^{2}, \Omega:=\left\{x: 0<x_{2}<d\right\}$ be a horizontal strip of width $d>0$. By $\gamma$, we denote a curve in $\Omega$ separated from $\partial \Omega$ by a fixed distance. Curve $\gamma$ is supposed to be $C^{3}$-smooth and to have no self-intersections. We consider two cases assuming that $\gamma$ is either an infinite curve or it is a finite closed curve. By $s$, we denote the arc length of $\gamma, s \in\left[-s_{*}, s_{*}\right]$, where $s_{*}$ is either finite or $s_{*}=+\infty$. If curve $\gamma$ is finite, we identify points $s=-s_{*}$ and $s=s_{*}$. By $\varrho=\varrho(s)$ we denote the vector function describing the curve $\gamma$. Since curve $\gamma$ is $C^{3}$-smooth, then $\varrho \in C^{3}\left[-s_{*}, s_{*}\right]$; for an infinite curve by $\left[-s_{*}, s_{*}\right]$ we mean $\mathbb{R}$. The above assumptions for $\gamma$ yield that this curve partitions domain $\Omega$ into two disjoint subdomains. The upper or exterior one is denoted by $\Omega_{+}$and the lower or interior subdomain is $\Omega_{-}$. By $B_{r}(a)$ we denote the ball in $\mathbb{R}^{2}$ of radius $r$ centered at $a$.

Let $\mathrm{IM}^{\varepsilon} \subseteq \mathbb{Z}$ be some set, and $s_{k}^{\varepsilon} \in\left[-s_{*}, s_{*}\right], k \in \mathrm{M}^{\varepsilon}$, be a set of points satisfying $s_{k}^{\varepsilon}<s_{k+1}^{\varepsilon}$. By $\omega_{k}, k \in \mathrm{IM}^{\varepsilon}$, we indicate a set of bounded domains in $\mathbb{R}^{2}$ having $C^{2}$-boundaries. We stress that these domains are not supposed to be simply connected. Denoting by $\varepsilon$ a small positive parameter, we define:

$$
\theta^{\varepsilon}:=\theta_{0}^{\varepsilon} \cup \theta_{1}^{\varepsilon}, \quad \theta_{i}^{\varepsilon}:=\bigcup_{k \in \mathbb{M}_{i}} \omega_{k}^{\varepsilon}, \quad i=0,1, \quad \omega_{k}^{\varepsilon}:=\left\{x: \varepsilon^{-1} \eta^{-1}(\varepsilon)\left(x-y_{k}^{\varepsilon}\right) \in \omega_{k}\right\}, \quad y_{k}^{\varepsilon}:=\varrho\left(s_{k}^{\varepsilon}\right),
$$

where $\mathbf{M}_{0}^{\varepsilon} \cap \mathbf{M}_{1}^{\varepsilon}=\emptyset, \mathbb{M}_{0}^{\varepsilon} \cup \mathbf{M}_{1}^{\varepsilon}=\mathbb{M}^{\varepsilon}$, and $\eta=\eta(\varepsilon)$ is a some function satisfying the inequality $0<\eta(\varepsilon) \leqslant 1$. We make the following assumptions.

(A1) There exist fixed numbers $0<R_{1}<R_{2}, b>1, L>0$ and points $x^{k} \in \omega_{k}, k \in \mathbb{I}^{\varepsilon}$, such that

$$
\begin{aligned}
& B_{R_{1}}\left(x^{k}\right) \subset \omega_{k} \subset B_{R_{2}}(0), \quad\left|\partial \omega_{k}\right| \leqslant L \quad \text { for each } k \in \mathbb{M}^{\varepsilon}, \\
& B_{b R_{2} \varepsilon}\left(y_{k}^{\varepsilon}\right) \cap B_{b R_{2} \varepsilon}\left(y_{i}^{\varepsilon}\right)=\emptyset \text { for each } i, k \in \mathbb{M}^{\varepsilon}, \quad i \neq k,
\end{aligned}
$$

and for all sufficiently small $\varepsilon$.

(A2) For $b$ and $R_{2}$ in $\mathrm{A} 1$ and $k \in \mathrm{IM}^{\varepsilon}$ there exists a generalized solution $X_{k}: B_{b_{*} R_{2}}(0) \backslash \omega_{k} \mapsto \mathbb{R}^{2}, b_{*}:=(b+1) / 2$, to the boundary value problem:

$$
\begin{gathered}
\operatorname{div} X_{k}=0 \quad \text { in } B_{b_{*} R_{2}}(0) \backslash \omega_{k}, \\
X_{k} \cdot \nu=-1 \quad \text { on } \partial \omega_{k}, \quad X_{k} \cdot \nu=\varphi_{k} \quad \text { on } \partial B_{b_{*} R_{2}}(0),
\end{gathered}
$$

belonging to $L_{\infty}\left(B_{b_{*} R_{2}}(0) \backslash \omega_{k}\right)$ and bounded in the sense of this space uniformly in $k \in \mathrm{I}^{\varepsilon}$. Here, $\nu$ is the outward normal to $\partial B_{b_{*} R_{2}}(0)$ and to $\partial \omega_{k}$, while $\varphi_{k}$ is a some function in $L_{\infty}\left(\partial B_{b_{*} R_{2}}(0)\right)$ satisfying:

$$
\int_{\partial B_{b_{*} R_{2}}(0)} \varphi_{k} d s=\left|\partial \omega_{k}\right| .
$$




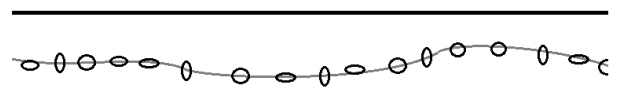

(a) Perforation along an infinite curve

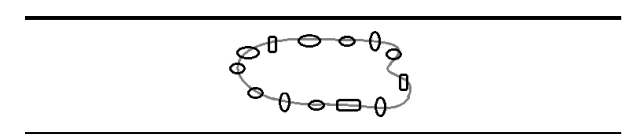

(b) Perforation along a closed curve

FIG. 1. Perforated domain

By $A_{i j}=A_{i j}(x), A_{i}=A_{i}(x), A_{0}=A_{0}(x)$ we denote functions satisfying the conditions:

$$
\begin{aligned}
& A_{i j}, A_{i} \in W_{\infty}^{1}(\Omega), \quad i, j=1,2, \quad A_{0} \in L_{\infty}(\Omega), \quad A_{j i}=A_{j i}, \\
& \sum_{i, j=1}^{2} A_{i j} z_{i} z_{j} \geqslant c_{2}|\xi|^{2}, \quad x \in \Omega, \quad z=\left(z_{1}, z_{2}\right) \in \mathbb{R}^{2}
\end{aligned}
$$

where $c_{2}$ is a positive constant independent of $x$ and $\xi$, and $A_{i j}, A_{0}$ are real-valued.

In the vicinity of $\gamma$ we introduce local coordinates $(s, \tau)$, where $\tau$ is the distance to a point measured along the normal $\nu^{0}$ to $\gamma$ which is inward for $\Omega_{-}$, and $s$, we remind, is the arc length of $\gamma$. Since the curvature of $\gamma$ is uniformly bounded, the coordinates $(s, \tau)$ are well-defined for $|\tau| \leqslant \tau_{0}, s \in \mathbb{R}$, where $\tau_{0}$ is a sufficiently small fixed positive number.

We denote by $\Omega^{\varepsilon}:=\Omega \backslash \theta^{\varepsilon}$ our perforated domain, cf. Fig. 1 . In this paper we study a singularly perturbed operator depending on $\varepsilon$ which we denote as $\mathcal{H}^{\varepsilon}$. It is introduced by the differential expression

$$
-\sum_{i, j=1}^{2} \frac{\partial}{\partial x_{i}} A_{i j} \frac{\partial}{\partial x_{j}}+\sum_{j=1}^{2} A_{j} \frac{\partial}{\partial x_{j}}-\frac{\partial}{\partial x_{j}} \overline{A_{j}}+A_{0}
$$

in $\Omega^{\varepsilon}$ subject to the Dirichlet condition on $\partial \Omega \cup \partial \theta_{0}^{\varepsilon}$ and to the Robin condition

$$
\left(\frac{\partial}{\partial N^{\varepsilon}}+a\right) u=0 \quad \text { on } \quad \partial \theta_{1}^{\varepsilon}, \quad \frac{\partial}{\partial N^{\varepsilon}}:=\sum_{i, j=1}^{2} A_{i j} \nu_{i}^{\varepsilon} \frac{\partial}{\partial x_{j}}+\sum_{j=1}^{2} \bar{A}_{j} \nu_{j}^{\varepsilon},
$$

where $\nu^{\varepsilon}=\left(\nu_{1}^{\varepsilon}, \nu_{2}^{\varepsilon}\right)$ is the inward normal to $\partial \theta_{1}^{\varepsilon}, a=a(x)$ is a function defined for $|\tau|<\tau_{0}$ and $a \in W_{\infty}^{1}(\{x:|\tau|<$ $\left.\left.\tau_{0}\right\}\right)$.

By $\mathfrak{a}^{\varepsilon}$ we denote the sesquilinear form:

$$
\begin{aligned}
\mathfrak{a}^{\varepsilon}(u, v):= & \sum_{i, j=1}^{2}\left(A_{i j} \frac{\partial u}{\partial x_{j}}, \frac{\partial v}{\partial x_{i}}\right)_{L_{2}\left(\Omega^{\varepsilon}\right)}+\sum_{j=1}^{2}\left(A_{j} \frac{\partial u}{\partial x_{j}}, v\right)_{L_{2}\left(\Omega^{\varepsilon}\right)} \\
& +\sum_{j=1}^{2}\left(u, A_{j} \frac{\partial v}{\partial x_{j}}\right)_{L_{2}\left(\Omega^{\varepsilon}\right)}+\left(A_{0} u, v\right)_{L_{2}\left(\Omega^{\varepsilon}\right)}
\end{aligned}
$$

in $L_{2}\left(\Omega^{\varepsilon}\right)$ on the domain $W_{2}^{1}\left(\Omega^{\varepsilon}\right)$. Rigorously, we introduce operator $\mathcal{H}^{\varepsilon}$ as the lower-semibounded self-adjoint operator in $L_{2}\left(\Omega^{\varepsilon}\right)$ associated with the closed lower-semibounded symmetric sesquilinear form $\mathfrak{h}^{\varepsilon}(u, v):=\mathfrak{a}^{\varepsilon}(u, v)+$ $(a u, v)_{L_{2}\left(\partial \theta_{1}^{\varepsilon}\right)}$ in $L_{2}\left(\Omega^{\varepsilon}\right)$ on $\stackrel{\circ}{W}_{2}^{1}\left(\Omega^{\varepsilon}, \partial \Omega \cup \partial \theta_{0}^{\varepsilon}\right)$. Hereafter, for any domain $Q \subset \mathbb{R}^{2}$ and any curve $S \subset Q$, by $\stackrel{\circ}{W}_{2}^{1}(Q, S)$, we denote the subspace of $W_{2}^{1}(Q)$ consisting of the functions with zero trace on $S$, and we let $\stackrel{\circ}{W}_{2}^{1}(Q):=\stackrel{\circ}{W}_{2}^{1}(Q, \partial Q)$. If else is not said, in what follows, all the differential operators are introduced in this way, i.e., they will be self-adjoint lower semibounded operators in $L_{2}(\Omega)$ or $L_{2}\left(\Omega^{\varepsilon}\right)$ associated with closed lower-semibounded symmetric sesquilinear form. For the sake of brevity, for each operator, we shall just write the differential expression with the boundary condition as well as the associated form.

Our main aim is to study the resolvent convergence and the spectrum's behavior of the operator $\mathcal{H}^{\varepsilon}$. To formulate our main results, we need additional notations.

By $\mathcal{H}_{\mathrm{D}}^{0}$ we denote the operator in $L_{2}(\Omega)$ with the differential expression (2.4) subject to the Dirichlet condition on $\gamma$ and $\partial \Omega$. The associated form is $\mathfrak{h}_{\mathrm{D}}^{0}(u, v):=\mathfrak{a}(u, v)$ in $L_{2}(\Omega)$ on $\stackrel{\circ}{W}_{2}^{1}(\Omega, \partial \Omega \cup \gamma)$, where form $\mathfrak{a}$ is introduced by expression (2.5), where $\Omega^{\varepsilon}$ is replaced by $\Omega$. By analogy with [46, Lem. 2.2], [47, Ch. IV, Sec. 2.2, 2.3], [48, Lem. 3.2] one can check that the domains of operator $\mathcal{H}_{\mathrm{D}}^{0}$ is given by the identity $\mathfrak{D}\left(\mathcal{H}_{\mathrm{D}}^{0}\right)=\stackrel{\circ}{W}_{2}^{1}(\Omega, \partial \Omega \cup \gamma) \cap W_{2}^{2}(\Omega \backslash \gamma)$. By i we denote the imaginary unit, and by $\|\cdot\|_{X \rightarrow Y}$ we denote the norm of an operator acting from a Banach space $X$ to a Banach space $Y$.

Now, we are ready to formulate our first main result.

Theorem 2.1. Let

and suppose (A1), (A2), and

$$
\varepsilon \ln \eta(\varepsilon) \rightarrow 0, \quad \varepsilon \rightarrow+0,
$$


(A3) There exists a constant $R_{3}>b R_{2}$ such that

$$
\left\{x:|\tau|<\varepsilon b R_{2}\right\} \subset \bigcup_{k \in \mathbb{M}_{0}^{\varepsilon}} B_{R_{3} \varepsilon}\left(y_{k}^{\varepsilon}\right), \quad \omega_{k}^{\varepsilon} \subset B_{R_{3} \varepsilon}\left(y_{k}^{\varepsilon}\right) \quad \text { for each } k \in \mathbb{M}_{0}^{\varepsilon} .
$$

Then, the estimate:

$$
\left\|\left(\mathcal{H}^{\varepsilon}-\mathrm{i}\right)^{-1}-\left(\mathcal{H}_{\mathrm{D}}^{0}-\mathrm{i}\right)^{-1}\right\|_{L_{2}(\Omega) \rightarrow W_{2}^{1}\left(\Omega^{\varepsilon}\right)} \leqslant C \varepsilon^{\frac{1}{2}}\left(|\ln \eta(\varepsilon)|^{\frac{1}{2}}+1\right)
$$

holds true, where $C$ is a positive constant independent of $\varepsilon$.

Let $\nu^{0}=\left(\nu_{1}^{0}, \nu_{2}^{0}\right)$ and

$$
\frac{\partial}{\partial N^{0}}:=\sum_{i, j=1}^{2} A_{i j} \nu_{i}^{0} \frac{\partial}{\partial x_{j}}
$$

By $[\cdot]_{\gamma}$ we indicate the jump of a function on $\gamma,[u]_{\gamma}=\left.u\right|_{\tau=+0}-\left.u\right|_{\tau=-0}$. Given a function $\beta=\beta(s)$ in $W_{\infty}^{1}(\gamma)$, we introduce operator $\mathcal{H}_{\beta}^{0}$ with differential expression (2.4) subject to the boundary conditions

$$
[u]_{\gamma}=0, \quad\left[\frac{\partial u}{\partial N^{0}}\right]_{\gamma}+\left.\beta u\right|_{\gamma}=0 .
$$

The associated form is $\mathfrak{h}_{\beta}^{0}(u, v):=\mathfrak{a}(u, v)+(\beta u, v)_{L_{2}(\gamma)}$ in $L_{2}(\Omega)$ on $\stackrel{\circ}{W}_{2}^{1}(\Omega)$. Again, by analogy with [46, Lem. 2.2], [47, Ch. IV, Sec. 2.2, 2.3], [48, Lem. 3.2], one can show that:

$$
\mathfrak{D}\left(\mathcal{H}_{\beta}^{0}\right)=\left\{u \in \stackrel{\circ}{W}_{2}^{1}(\Omega): u \in W_{2}^{2}\left(\Omega_{ \pm}\right) \text {and (2.8) is satisfied }\right\} .
$$

If $\beta=0$, instead of $\mathcal{H}_{0}^{0}$ we shall simply write $\mathcal{H}^{0}$. As one can see, in this case there is no boundary condition on $\gamma$ and the domain of $\mathcal{H}^{0}$ is $\mathfrak{D}\left(\mathcal{H}^{0}\right)=\stackrel{\circ}{W}_{2}^{1}(\Omega) \cap W_{2}^{2}(\Omega)$.

In the next theorem, we deal with the case when the perturbed operator involves the Dirichlet condition at least on a part of $\partial \theta^{\varepsilon}$ but in contrast to (2.6), the function $\varepsilon \ln \eta(\varepsilon)$ converges either to a non-zero constant or to infinity.

Theorem 2.2. Suppose (A1), (A2), let:

$$
\frac{1}{\varepsilon \ln \eta(\varepsilon)} \rightarrow-\rho, \quad \varepsilon \rightarrow+0
$$

and set $\mathrm{M}_{0}^{\varepsilon}$ be non-empty. For $b$ and $R_{2}$ in $A l$ and $s \in \mathbb{R}$ we denote:

$$
\alpha^{\varepsilon}(s):=\left\{\begin{array}{cc}
\frac{\pi}{b R_{2}}, & \left|s-s_{k}^{\varepsilon}\right|<b R_{2} \varepsilon, \quad k \in \mathbf{M}_{0}^{\varepsilon}, \\
0, & \text { otherwise. }
\end{array}\right.
$$

Suppose also that:

(A4) There exists a function $\alpha=\alpha(s)$ in $W_{\infty}^{1}(\gamma)$ and a function $\varkappa=\varkappa(\varepsilon), \varkappa(\varepsilon) \rightarrow+0, \varepsilon \rightarrow+0$, such that for all sufficiently small $\varepsilon$ the estimate:

$$
\sum_{q \in \mathbb{Z}} \frac{1}{|q|+1}\left|\int_{n}^{n+\ell}\left(\alpha^{\varepsilon}(s)-\alpha(s)\right) \mathrm{e}^{-\frac{\mathrm{i} q}{2 \pi \ell}(s-n)} d s\right|^{2} \leqslant \varkappa^{2}(\varepsilon)
$$

is valid, where $n=-s_{*}, \ell=2 s_{*}$, if $\gamma$ is a finite curve, and $n \in \mathbb{Z}, \ell=1$, if $\gamma$ is an infinite curve. In the latter case estimate (2.10) is supposed to hold uniformly in $n$.

We denote:

Then the estimates:

$$
\beta:=\alpha \frac{(\rho+\mu)}{A_{11} A_{22}-A_{12}^{2}}, \quad \beta_{0}:=\alpha \frac{\rho}{A_{11} A_{22}-A_{12}^{2}}, \quad \mu(\varepsilon):=-\frac{1}{\varepsilon \ln \eta(\varepsilon)}-\rho .
$$

$$
\begin{aligned}
& \left\|\left(\mathcal{H}^{\varepsilon}-\mathrm{i}\right)^{-1}-\left(\mathcal{H}_{\beta}^{0}-\mathrm{i}\right)^{-1}\right\|_{L_{2}(\Omega) \rightarrow L_{2}\left(\Omega^{\varepsilon}\right)} \leqslant C\left(\varepsilon^{\frac{1}{2}}+\varkappa(\varepsilon)\right) \\
& \left\|\left(\mathcal{H}^{\varepsilon}-\mathrm{i}\right)^{-1}-\left(\mathcal{H}_{\beta_{0}}^{0}-\mathrm{i}\right)^{-1}\right\|_{L_{2}(\Omega) \rightarrow L_{2}\left(\Omega^{\varepsilon}\right)} \leqslant C\left(\varepsilon^{\frac{1}{2}}+\varkappa(\varepsilon)+\mu(\varepsilon)\right)
\end{aligned}
$$

hold true, where $C$ is a positive constant independent of $\varepsilon$. There exists an explicit function $W^{\varepsilon}$ (see (6.5) in [1]) such that the estimate:

$$
\left\|\left(\mathcal{H}^{\varepsilon}-\mathrm{i}\right)^{-1}-\left(1-W^{\varepsilon}\right)\left(\mathcal{H}_{\beta}^{0}-\mathrm{i}\right)^{-1}\right\|_{L_{2}(\Omega) \rightarrow W_{2}^{1}\left(\Omega^{\varepsilon}\right)} \leqslant C\left(\varepsilon^{\frac{1}{2}}+\varkappa(\varepsilon)(\rho+\mu(\varepsilon))\right),
$$

is valid, where $C$ is a positive constant independent of $\varepsilon$. If $\rho=0$, the estimate

$$
\left\|\left(\mathcal{H}^{\varepsilon}-\mathrm{i}\right)^{-1}-\left(\mathcal{H}^{0}-\mathrm{i}\right)^{-1}\right\|_{L_{2}(\Omega) \rightarrow W_{2}^{1}\left(\Omega^{\varepsilon}\right)} \leqslant C\left(\varepsilon^{\frac{1}{2}}+\mu^{\frac{1}{2}}(\varepsilon)\right)
$$

holds true, where $C$ is a positive constant independent of $\varepsilon$.

The next two theorems concern the case when $\mathrm{M}_{0}^{\varepsilon}$ is empty, i.e., the perturbed operator involves just the Robin condition on $\partial \theta^{\varepsilon}$. 
Theorem 2.3. Suppose (Al), (A1), let set $\mathrm{M}_{0}^{\varepsilon}$ be empty and either $a \equiv 0$ or $\eta(\varepsilon) \rightarrow 0, \varepsilon \rightarrow+0$. Then the estimate

$$
\left\|\left(\mathcal{H}^{\varepsilon}-\mathrm{i}\right)^{-1}-\left(\mathcal{H}^{0}-\mathrm{i}\right)^{-1}\right\|_{L_{2}(\Omega) \rightarrow W_{2}^{1}\left(\Omega^{\varepsilon}\right)} \leqslant C \eta(\varepsilon)|\ln \eta(\varepsilon)|^{\frac{1}{2}},
$$

holds true, if $a \not \equiv 0, \eta \rightarrow+0$, and

$$
\left\|\left(\mathcal{H}^{\varepsilon}-\mathrm{i}\right)^{-1} f-\left(\mathcal{H}^{0}-\mathrm{i}\right)^{-1} f\right\|_{L_{2}(\Omega) \rightarrow W_{2}^{1}\left(\Omega^{\varepsilon}\right)} \leqslant C \varepsilon^{\frac{1}{2}} \eta(\varepsilon)\left(|\ln \eta(\varepsilon)|^{\frac{1}{2}}+1\right),
$$

if $a \equiv 0$. Here $C$ is a positive constant independent of $\varepsilon$.

Theorem 2.4. Suppose (A1), (A2), let $\eta=$ const, set $\mathrm{IM}_{0}^{\varepsilon}$ be empty. For b and $R_{2}$ in (A1) we denote:

$$
\alpha^{\varepsilon}(s):=\left\{\begin{array}{cl}
\frac{\left|\partial \omega_{k}\right| \eta}{2 b R_{2}}, & \left|s-s_{k}^{\varepsilon}\right|<b R_{2} \varepsilon, \quad k \in \mathbb{M}^{\varepsilon}, \\
0, & \text { otherwise. }
\end{array}\right.
$$

Suppose also that:

(A5) There exists a function $\alpha=\alpha(s)$ in $W_{\infty}^{1}(\gamma)$ and a function $\varkappa=\varkappa(\varepsilon), \varkappa(\varepsilon) \rightarrow+0, \varepsilon \rightarrow+0$, such that for all sufficiently small $\varepsilon$ the estimates (2.10) are valid.

Then the estimate:

$$
\left\|\left(\mathcal{H}^{\varepsilon}-\mathrm{i}\right)^{-1}-\left(\mathcal{H}_{\alpha \mathrm{a}}^{0}-\mathrm{i}\right)^{-1}\right\|_{L_{2}(\Omega) \rightarrow W_{2}^{1}\left(\Omega^{\varepsilon}\right)} \leqslant C\left(\varepsilon^{\frac{1}{2}}+\varkappa(\varepsilon)\right)
$$

holds true, where $C$ is a positive constant independent of $\varepsilon$.

Let us discuss the main results. Assumption (A1) says that the sizes of holes are of the same order and there is a minimal distance between them. This is a very natural assumption for the perforation. At the same time, no periodicity for the perforation is assumed. Moreover, since set $\mathrm{IM}^{\varepsilon}$ is arbitrary, we do not need to assume that it is infinite, and for instance, the number of holes can be finite. In the latter case, by choosing an appropriate set $\mathrm{IM}^{\varepsilon}$, we can even get the situation when the distances between the holes are not small, but finite. In this situation one can still apply Theorems $2.2-$ 2.4. Theorem 2.1 is valid only in the case when the holes with Dirichlet condition are distributed quite densely in order to satisfy Assumption A3.

Assumption (A2) is a restriction for the geometry of boundaries $\partial \omega_{k}$. We first stress that problem (2.1) can be rewritten to the Neumann problem for the Laplace equation by letting $X_{k}=\nabla V_{k}$. Then identity (2.2) is the solvability condition and this is the only restriction for $\varphi_{k}$ we suppose. Problem (2.1) is solvable for each fixed $k$ and it is solution belongs to $L_{\infty}\left(B_{b_{*} R_{2}}(0) \backslash \omega_{k}\right)$. And we assume that the norm $\left\|X_{k}\right\|_{L_{\infty}\left(B_{b_{*} R_{2}}(0) \backslash \omega_{k}\right)}$ is bounded uniformly in $k$.

According to Theorem 2.1, if the sizes of the holes are not too small (cf. (2.6)) and the holes with the Dirichlet condition are, roughly speaking, distributed "uniformly" (Assumption (A3)), the homogenized operator is subject to the Dirichlet condition on $\gamma$ and we have the norm resolvent condition in the sense of the operator norm $\|\cdot\|_{L_{2}(\Omega) \rightarrow L_{2}\left(\Omega^{\varepsilon}\right)}$. As one can see, relation (2.6) admits the situation when the sizes of the holes are much smaller than the distances between them (for instance, $\eta(\varepsilon)=\varepsilon^{\alpha}, \alpha=$ const $>0$ ), but nevertheless the homogenized operator is still subject to the Dirichlet condition on $\gamma$. This phenomenon is close to a similar one for the operators with frequent alternation of boundary conditions, cf. [28, 29,49].

If the function $\varepsilon \ln \eta(\varepsilon)$ goes to a constant or to infinity as $\varepsilon \rightarrow+0$ and there are holes with the Dirichlet condition, the homogenized operator has boundary condition (2.8) on $\gamma$, see Theorem 2.2. This boundary condition describes a delta-interaction on $\gamma$, see, for instance, [50, App. K, Sec. K.4.1], and the similar situation holds for the problems with frequent alternation of boundary conditions with the Dirichlet conditions on exponentially small parts of the boundary, cf. [26-28, 49]. The norm resolvent convergence holds in the sense of the operator norm $\|\cdot\|_{L_{2}(\Omega) \rightarrow L_{2}\left(\Omega^{\varepsilon}\right)}$ only. To improve the norm, one has either to employ the boundary corrector, see (2.13), or to assume additionally $\rho=0$, see (2.14). We observe that according to Assumption (A4), coefficient $\beta$ in boundary condition (2.8) for the homogenized operator depends only on the distribution of the points $s_{k}^{\varepsilon}$ and there is no dependence on the geometries of the holes. There are also no special restrictions for part $\partial \theta_{0}^{\varepsilon}$ with the Dirichlet condition. For instance, the number of holes in $\partial \theta_{0}^{\varepsilon}$ can be finite or infinite and the distribution of this set can be very arbitrary.

If the perturbed operator has no Dirichlet condition on $\partial \theta^{\varepsilon}$, the homogenized operator has either condition (2.8) on $\gamma$ (Theorem 2.4) or even no condition (Theorem 2.3). In both cases we again have the norm resolvent convergence in the operator norm $\|\cdot\|_{L_{2}(\Omega) \rightarrow W_{2}^{1}\left(\Omega^{\varepsilon}\right)}$. In Theorem 2.3 we need no additional restrictions thanks to the assumption $\eta(\varepsilon) \rightarrow+0$ or $a \equiv 0$. In Theorem $2.4 \eta$ is constant and because of this we introduce Assumption (A5). Its means that the lengths of $\partial \omega_{k}$ should be distributed rather smoothly to satisfy (2.10). We stress that the coefficient $\beta$ in (2.8) for the homogenized operator depends both on the distribution of the holes and the sizes of their boundaries.

Let us also discuss assumptions (A4) and (A5). This is in fact the same assumption but adapted for two different cases. The sum in the left hand side of (2.10) is nothing but the norm in $W_{2}^{-\frac{1}{2}}(0, \ell)$. This estimate obviously holds true for a periodic perforation. As an example of a non-periodic perforation, we can mention the situation when we start with a strictly periodic perforation along an infinite curve but then we change the geometry and locations of a part of holes so that the total number of deformed holes associated with each segment $s \in(q, q+1), q \in \mathbb{Z}$, is relatively small in 
comparison with unchanged holes. Then inequality (2.10) is still true. Moreover, our conjecture is that Assumptions (A4) and (A5) can not be improved or omitted once we want to have a norm resolvent convergence.

About the sharpness of the estimates for the rate of convergences, we observe that many of these estimates are order sharp, i.e., the smallness order can not be improved. At the same time, the study of the sharpness is an independent problem that requires a completely different approach in comparison with the technique we employ in the proofs of Theorems 2.1-2.4. The sharpness of the estimates are proved in Section 8 of [1].

Our final main result describes the convergence of the spectrum of $\mathcal{H}^{\varepsilon}$.

Theorem 2.5. Under the hypotheses of Theorems 2.1-2.4 the spectrum of perturbed operator $\mathcal{H}^{\varepsilon}$ converges to that of the corresponding homogenized operator. Namely, if $\lambda$ is not in the spectrum of the homogenized operator, for sufficiently small $\varepsilon$, the same is true for the perturbed operator. And if $\lambda$ is in the spectrum of the homogenized operator, for each $\varepsilon$, there exists $\lambda_{\varepsilon}$ in the spectrum of the perturbed operator such that $\lambda_{\varepsilon} \rightarrow \lambda$ as $\varepsilon \rightarrow+0$.

We note that this theorem is not implied immediately by Theorems 2.1-2.4. Despite these theorems state convergence of the perturbed resolvent to a homogenized one in the norm sense, the norm is $\varepsilon$-dependent. Nevertheless, this makes no serious troubles and in the proof of Theorem 2.5 it is proved a simple trick to overcome this difficulty.

\section{References}

[1] Borisov D., Cardone G., Durante T. Homogenization and uniform resolvent convergence for elliptic operators in a strip perforated along a curve. Proc. R. Soc.Edingurgh Sect. A, 2016, 146(6), P. 115-1158.

[2] Belyaev A.G. Averaging of a mixed boundary value problem for the Poisson equation in a domain perforated along the boundary. Russ. Math. Surv., 1990, 45, P. 140.

[3] Chechkin G.A., Chechkina T.A., D'Apice C., De Maio U. Homogenization in domains randomly perforated along the boundary. Discr. Cont. Dynam. Syst., Ser. B., 2009, 12, P. 713-730.

[4] Chechkin G.A., Koroleva Yu.O., Meidell A., Persson L.-E. On the Friedrichs Inequality in a Domain Perforated Aperiodically along the Boundary. Homogenization Procedure. Asymptotics for Parabolic Problems. Russ. J. Math. Phys., 2009, 16, P. 1-16.

[5] Gómez D., Lobo M., Pérez M.E., Shaposhnikova T.A. Averaging of variational inequalities for the Laplacian with nonlinear restrictions along manifolds. Appl. Anal., 2013, 92, P. 218-237.

[6] Gómez D., Pérez M.E., Shaposhnikova T.A. On homogenization of nonlinear Robin type boundary conditions for cavities along manifolds and associated spectral problems. Asymptot. Anal., 2012, 80, P. 289-322.

[7] Lobo M., Oleinik O.A., Pérez M.E., Shaposhnikova T.A. On homogenizations of solutions of boundary value problems in domains, perforated along manifolds. Ann. Sc. Norm. Super. Pisa Cl. Sci., 1997, 25(4), P. 611-629.

[8] Lobo M., Oleinik O.A., Pérez M.E., Shaposhnikova T.A. On boundary-value problems in domains perforated along manifolds. Russ. Math. Surv., 1997, 52, P. 838-839.

[9] Birman M.Sh. On the averaging procedure for periodic operators in a neighborhood of an edge of an internal gap. St. Petersburg Math. J., 2004, 15, P. 507-513.

[10] Birman M.Sh., Suslina T.A. Homogenization with corrector term for periodic elliptic differential operators. St. Petersburg Math. J., 2006, 17, P. 897-973.

[11] Birman M.Sh., Suslina T.A. Homogenization with corrector for periodic differential operators. Approximation of solutions in the Sobolev class $H^{1}\left(\mathbb{R}^{d}\right)$. St. Petersburg Math. J., 2007, 18, P. 857-955.

[12] Suslina T.A. Homogenization with corrector for a stationary periodic Maxwell system. St. Petersburg Math. J., 2008, 19, P. $455-494$.

[13] Suslina T.A. Homogenization in Sobolev class $H^{1}\left(\mathbb{R}^{d}\right)$ for periodic elliptic second order differential operators including first order terms. St. Petersburg Math. J., 2011, 22, P. 81-162.

[14] Suslina T.A., Kharin A.A. Homogenization with corrector for a periodic elliptic operator near an edge of inner gap. J. Math. Sci., 2009, 159, P. 264-280.

[15] Cardone G., Pastukhova S.E., Zhikov V.V. Some estimates for nonlinear homogenization. Rend. Accad. Naz. Sci. XL Mem. Mat. Appl., 2005, 29, P. 101-110.

[16] Pastukhova S.E. Some Estimates from Homogenized Elasticity Problems. Dokl. Math., 2006, 73, P. 102-106.

[17] Pastukhova S.E., Tikhomirov R.N. Operator Estimates in Reiterated and Locally Periodic Homogenization. Dokl. Math., 2007, 76, P. 548-553.

[18] Zhikov V.V. On operator estimates in homogenization theory. Dokl. Math., 2005, 72, P. 534-538.

[19] Zhikov V.V. Spectral method in homogenization theory. Proc. Steklov Inst. Math., 2005, 250, P. 85-94.

[20] Zhikov V.V. Some estimates from homogenization theory. Dokl. Math., 2006, 73, P. 96-99.

[21] Griso G. Error estimate and unfolding for periodic homogenization. Asymptot. Anal., 2004, 40, P. 269-286.

[22] Griso G. Interior error estimate for periodic homogenization. C. R. Acad. Sci. Paris Ser. I Math., 2005, 340, P. 251-254.

[23] Kenig C.E., Lin F., Shen Z. Convergence rates in $L^{2}$ for elliptic homogenization problems. Arch. Rat. Mech. Anal., 2012, 203, P. $1009-1036$.

[24] Kenig C.E., Lin F., Shen Z. Periodic homogenization of green and neumann functions. Comm. Pure Appl. Math., 2014, 67, P. 1219-1262.

[25] Borisov D., Bunoiu R., Cardone G. Homogenization and asymptotics for a waveguide with an infinite number of closely located small windows. J. Math. Sci., 2011, 176, P. 774-785.

[26] Borisov D., Bunoiu R., Cardone G. On a waveguide with frequently alternating boundary conditions: homogenized Neumann condition. Ann. Henri Poincaré, 2010, 11, P. 1591-1627.

[27] Borisov D., Bunoiu R., Cardone G. On a waveguide with an infinite number of small windows. Compt. Rend. Math., 2011, 349, P. 53-56.

[28] Borisov D., Bunoiu R., Cardone G. Waveguide with non-periodically alternating Dirichlet and Robin conditions: homogenization and asymptotics. Z. Angew. Math. Phys., 2013, 64, P. 439-472.

[29] Borisov D., Cardone G. Homogenization of the planar waveguide with frequently alternating boundary conditions. J. Phys. A., 2009,42 , id 365205.

[30] Borisov, D., Cardone, G.: Complete asymptotic expansions for the eigenvalues of the Dirichlet Laplacian in thin three-dimensional rods, ESAIM: COCV 17 (2011), 887-908.

[31] Borisov D., Cardone G. Planar waveguide with "twisted" boundary conditions. Small width, J. Math. Phys., 2012, 53, 023503.

[32] Borisov D., Cardone G. Spectra of operator pencils with small PT-symmetric periodic perturbation. ESAIM: COCV, 2020, 26, 2019070. 
[33] Borisov D., Cardone G., Checkhin G., Koroleva Yu. On elliptic operators with Steklov condition perturbed by Dirichlet condition on a small part of boundary. Calc. Var. Part. Diff. Eq., 2021, 60, P. 48.

[34] Cardone G., Khrabustovskyi A. Neumann spectral problem in a domain with very corrugated boundary, J. Diff. Eq., $2015,259(6)$, P. $2333-2367$.

[35] Cardone G., Khrabustovskyi A. $\delta^{\prime}$-interaction as a limit of a thin Neumann waveguide with transversal window. J. Math. Anal. Appl., 2019, 473(2), P. $1320-1342$.

[36] Cardone G., Khrabustovskyi A. Spectrum of the Laplacian on a domain perturbed by small resonators, arXiv: 1808.05402.

[37] Cardone G., Minutolo V., Nazarov S.A. Gaps in the essential spectrum of periodic elastic waveguides. Z. Angew. Math. Mech., 2009, 89(9), P. 729-741.

[38] Cardone G., Nazarov S.A., Sokolowski. Asymptotics of solutions of the Neumann problem in a domain with closely posed components of the boundary. Asympt. Anal., 2009, 62, P. 41-88.

[39] Cardone G., Nazarov S.A., Taskinen J. Spectra of open waveguides in periodic media. J. Functional Anal., $2015,269(8)$, P. $2328-2364$.

[40] Aiyappan S., Cardone G., Perugia C., Prakash R. Homogenization of a nonlinear monotone problem in a locally periodic domain via unfolding method. Nonlinear Analysis: Real World Applications, 2022, 66, 103537.

[41] Borisov D., Cardone G., Faella L., Perugia C. Uniform resolvent convergence for a strip with fast oscillating boundary. J. Diff. Equ., 2013, 255, P. 4378-4402.

[42] Nazarov S.A. Dirichlet problem in an angular domain with rapidly oscillating boundary: Modeling of the problem and asymptotics of the solution. St. Petersburg Math. J., 2008, 19, P. 297-326.

[43] Olejnik O.A., Shamaev A.S., Yosifyan G.A. Mathematical Problems in Elasticity and Homogenization. Stud. Math. Appl., 1992, 26. North-Holland, Amsterdam.

[44] Marchenko V.A., Khruslov E.Ya. Boundary Value Problems in Domains with Fine-Grained Boundary. Naukova Dumka, Kiev, 1974. (in Russian)

[45] Cardone G., Panasenko G.P., Sirakov Y. Asymptotic analysis and numerical modeling of mass transport in tubular structures. Math. Models Meth. Appl. Sc., 2010, 20(4), P. 1-25.

[46] Borisov D. Asymptotics for the solutions of elliptic systems with fast oscillating coefficients. St. Petersburg Math. J., 2009, 20, P. 175-191.

[47] Mikhajlov V.P. Partial Differential Equations. Mir Publishers, Moscow, 1978.

[48] Borisov D., Krejčiřık D. P T -symmetric waveguide. Integr. Equat. Oper. Th., 2008, 62, P. 489-515.

[49] Chechkin G.A. Averaging of boundary value problems with singular perturbation of the boundary conditions. Russ. Acad. Sci. Sb. Math., 1994, 79, P. 191-220.

[50] Albeverio S., Gesztesy F., Høegh-Krohn R., Holden H. Solvable models in quantum mechanics, 2nd ed. AMS Chelsea Publishing, Providence, Rhode Island, 2005.

Submitted 28 January 2022; revised 31 January 2022; accepted 2 February 2022

Information about the authors:

Giuseppe Cardone - Universita di Napoli "Federico II", Dipartimento di Matematica e Applicazioni "R. Caccioppoli", Via Cintia, Napoli, Italy; giuseppe.cardone@unina.it

Tiziana Durante - University of Salerno, Department of Information and Electrical Engineering and Applied Mathematics, Via Ponte Don Melillo, 1, 84084, Fisciano (SA), Italy; tdurante@unisa.it

Conflict of interest: the authors declare no conflict of interest. 